\title{
Prevalence of Ocular Surface Disorder and Dry Eye in Patients with Glaucoma using Topical Antiglaucoma Medications
}

\author{
Santosh Yadawrao Ingle ${ }^{1}$, Saswati Biswas ${ }^{2}$ \\ ${ }^{1}$ Consultant Ophthalmologist, Swara I Care Hospital, Amravati, Maharashtra, India. \\ ${ }^{2}$ Junior Resident, Susrut Eye Foundation and Research Centre, Kolkata West Bengal, India.
}

Corresponding Author: Santosh Yadawrao Ingle

\begin{abstract}
Background: The main aim of the study is to establish the prevalence of ocular surface disorders in glaucoma patients treated with topical antiglaucoma medications.

Methods: Investigation of 50 eyes of 25 patients has been performed using two or more antiglaucoma medications for six months, and compared with 50 eyes of 25 normal subjects without any ocular problem. After one year each patient was evaluated by Schirmer's (SCH) testI Tear break-up time (TBUT), Corneal staining score.
\end{abstract}

Results: The mean values in the glaucoma cases and control groups respectively were as follows: Schirmer's test-1 $(7.63 \pm 2.64 \mathrm{~mm} /$ $12.86 \pm 1.93 \mathrm{~mm} ; \mathrm{p}=0.001)$, Tear breakup time (9.44 $2 \pm .76 \mathrm{sec} / 11.8 \pm 1.88 \mathrm{sec} ; \mathrm{p}=0.001)$, Corneal staining score $(5.7 \pm 2.33 / 1.1 \pm 0.58$; $\mathrm{p}=0.001$ ).

Conclusion: Patients on antiglaucoma therapy have a greater prevalence of ocular surface disease and dry eye. The causative factors were medications with preservatives and longer treatment duration.

Keywords: Antiglaucoma medication, dry eye, ocular surface diseases (OSD), Schirmer's (SCH) test, Tear breakup time, Corneal staining score.

\section{INTRODUCTION}

Glaucoma is the second leading cause of blindness worldwide. The majority of patients diagnosed with glaucoma are benefitted from the treatment with intraocular pressure (IOP) lowering agent to achieve the target level in the initial stage of the disease.

Ocular surface side effects occur due to chronic long-term use of antiglaucoma medication. Instillation of topical antiglaucoma drops for a period of three or more months has been found to cause significant subclinical inflammation which has been detected as increased expression of HLA-DR on conjunctival epithelial cells ${ }^{1}$. Pro-inflammatory cytokines secreted by conjunctival cells causing epithelial cells damage, cells death by apoptosis, detergent effect on the lipid layer and decrease in goblet cell density, ultimately decreasing the tear film ${ }^{2-4}$. Clinical studies showed that the long-term use of topical drugs may induce ocular discomfort, tear film instability, conjunctival inflammation, subconjunctival fibrosis, corneal surface impairment and potential risk of failure of glaucoma surgery ${ }^{5,6}$. Ocular surface disease (OCD) demonstrates an overall prevalence of $42 \%$ (range 20-59\%) in glaucoma, which is severe in $36 \%$ (range 14-66\%) ${ }^{5}$. Benzalkonium chloride (BAC, quaternary ammonium compound), the most commonly used preservative in topical antiglaucoma drugs, which is retained in the ocular tissues for as long as $168 \mathrm{hrs}$ after application ${ }^{7}$. BAC promotes the activation of lipoxygenase, synthesis \& secretion of eicosanoids, inflammatory molecules and cytokines like interleukin (IL)-1, tumor 
necrosis factor, IL-8, IL-10, resulting in irritation, delayed hypersensitivity and allergic reaction ${ }^{8}$.

\section{MATERIALS \& METHODS}

The study was a prospective randomized trial conducted at Susrut Eye Foundation \& Research Centre, Kolkata during the duration of April 2018 to April 2019 and was conducted in accordance with the tenets of the Declaration of Helsinki.

In the study, subjects were divided into two groups. Group 1: 50 eyes of 25 patients using two antiglaucoma medications for at least 6 months. Group 2: 50 eyes of 25 normal subjects without any ocular problem.

\section{Inclusion criteria:}

1. Patients starting two antiglaucoma medications and never used these drugs before enrolling in this study.

2. Duration for taking medicines at least six months.

\section{Exclusion criteria:}

1. History of severe ocular trauma.

2. Past history of intraocular surgery or any laser treatment

3. Current use of contact lenses.

4. Presence of eyelid and eyelash deformity.

5. History of recent ocular inflammation or infection.

6. Previous or current use of any ocular medication including artificial tear therapy, any systemic treatment known to affect tear secretion like autoimmune diseases.

Follow up: All participants underwent a complete anterior segment evaluation, IOP measurement by Goldmann applanation tonometry, viewing iridocorneal angle, optic disc evaluation, visual field examination and tear function test (Schirmer's test-1, Tear breakup time, Corneal staining score) before the study. Then repeat tear function tests were performed at $1^{\text {st }}, 3^{\text {rd }}, 6^{\text {th }}$ and $12^{\text {th }}$ month.

Tests \& Studies: Schirmer's test-1 with no topical anesthesia was performed using Schirmer's paper strip placed in the lateral lower conjunctival sac. The paper strip was removed after 5 minutes, the length of the moistened area has been recorded. Tear quality was measured with Tear breakup test. A drop of sodium fluorescein dye was instilled and the average interval between the last complete blink and the appearance of the first dye spot on the pre-corneal film was calculated under cobalt blue filtered light. Corneal fluorescein staining was examined with cobalt blue illumination, lissamine green was then instilled and interpalpebral conjunctival staining of temporal \& nasal conjunctiva was graded by using the Oxford Score 6 points Scale (from $0-5)$.

Antiglaucoma medicines timolol, brimonidine, latanoprost and brimonidine have been used for 50 patients of Group 1 as per Table 1.

Table 1. List of antiglaucoma medicines with doses received by 50 enrolled eyes in antiglaucoma therapy group (Group 1).

\begin{tabular}{|l|l|l|}
\hline Number of eyes & Name of drugs & Dose \\
\hline 10 & Combination of $0.5 \%$ timolol and $0.2 \%$ brimonidine & One drop twice daily \\
\hline 8 & Combination of $0.005 \%$ latanoprost and $0.2 \%$ brimonidine & One drop twice daily \\
\hline 32 & Combination of $0.5 \%$ timolol and $0.005 \%$ latanoprost & One drop twice daily \\
\hline
\end{tabular}

\section{STATISTICAL ANALYSIS}

Statistical analysis was done using the program SPSS version 15. Quantitative variables (expressed as mean, standard deviation, range) were compared between the antiglaucoma medication group \& control group by using sample t- test with a
$\mathrm{P}$ value $<0.05$ considered as statistically significant.

\section{RESULTS}

One hundred patients participated in this study, of those 50 were on antiglaucoma therapy, and 50 were healthy subjects. The mean age of the participants of the 
Santosh Yadawrao Ingle et.al. Prevalence of ocular surface disorder and dry eye in patients with glaucoma using topical antiglaucoma medications.

glaucoma group who completed the study was 49-50 years, ranging from 22-75 years and for the control group 40-68 ranging from 26-65 years as per Table 2.

Table 2. Demographic characteristics of eyes on study

\begin{tabular}{|l|l|l|l|}
\hline Demographic data & Antiglaucoma Therapy group & Control group & P- value \\
\hline Number of eyes & 50 & 50 & \\
\hline Gender (M/F) & $29 / 21$ & $38 / 12$ & 0.056 \\
\hline Mean age & $49-52$ (range22-75) & $40-68$ (range 26-65) & 0.031 \\
\hline
\end{tabular}

The result of $\mathrm{SCH}$ I for the antiglaucoma therapy group (Group 1) was $7.63 \pm 2.64 \mathrm{~mm}$ (range 3-12), and that for the controls (Group 1) was $12.86 \pm 1.93$ (range 10-16) mm. A statistically significant difference was found between the two groups $(\mathrm{p}=0.001)$. In our study, we found that the Schirmer test values of the antiglaucoma therapy group patients were lower than those of the controls. Tear breakup time of Group 1 was $9.44 \pm$ $2.76 \mathrm{~mm}$ (range 7.1-15.2), and that for Group 1 was $11.80 \pm 1.88$ (range 9-15.6) $\mathrm{mm}$ with $\mathrm{p}$-value 0.001 . A significant difference was observed in the corneal staining score between two groups with pvalue of 0.001 .

Table 3. SCH I (mm) values and tear breakup time (TBUT) of the tear film (second) according to the groups during the beginning, 1st month, 3rd month, 6th month and 12th month in Group 1.

\begin{tabular}{|l|l|l|l|l|l|}
\hline Ocular surface evaluation tests & Pre study & $\mathbf{1}^{\text {st }}$ month & $\mathbf{3}^{\text {rd }}$ month & $\mathbf{6}^{\text {th }}$ month & $\mathbf{1 2}^{\text {th }}$ month \\
\hline Schirmer's test & $23.7 \pm 2.8$ & $12.15 \pm 3.1$ & $8.03 \pm 2.5$ & $6.24 \pm 1.57$ & $5.24 \pm 2.64$ \\
\hline Tear breakup time & $15.68 \pm 2.62$ & $15.23 \pm 1.58$ & $10.02 \pm 0.01$ & $9.02 \pm 1.01$ & $7.10 \pm 1.01$ \\
\hline Corneal staining score & 0 & $2.1 \pm 1.01$ & $3.02 \pm 1.04$ & $5.01 \pm 1.04$ & $9.01 \pm 1.03$ \\
\hline
\end{tabular}

Table 4. Ocular evaluation tests in antiglaucoma therapy group versus control group.

\begin{tabular}{|l|l|l|l|}
\hline Ocular evaluation tests & Antiglaucoma therapy group & Control group & P- value \\
\hline Schirmer's test & $7.63 \pm 2.64($ range $3-12)$ & $12.86 \pm 1.93($ range $10-16)$ & 0.001 \\
\hline Tear breakup time & $9.44 \pm 2.76($ range $7.1-15.20)$ & $11.80 \pm 1.88($ range $9-15.26)$ & 0.001 \\
\hline Corneal staining score & $5.7 \pm 2.33$ (range 2-9) & $1.1 \pm 0.05($ range $0-2)$ & 0.001 \\
\hline
\end{tabular}

\section{DISCUSSION}

Altered epithelial barrier function leads to exposure of corneal nerve ending to environmental stimuli, causing irritation, unstable tear film; resulting in ocular surface epithelial changes. The prevalence of ocular disease is higher in patients using BAC-containing antiglaucoma medication. Our study also found a similar prevalence of dry eye in $66 \%$ of our cases. Recent studies have shown that the exclusive use of preservative-free medication can reduce the sign of ocular surface alteration, compared with medicines with high BAC levels. Our study shows that, the time period rather than the quantity of drugs plays an important role in the progression of ocular surface disease (OCD). OCDs with dry eye can be a major reason for the loss of compliance in patients on antiglaucoma therapy. Long-term adherence with glaucoma treatment may be associated with the type of medication with higher adherence to prostaglandin analogs than beta blockers ${ }^{8}$, patient's beliefs, disease characteristics and satisfaction with treatment. Identifying and treatment of dry eye in patients with antiglaucoma medication can prove to be beneficial in long-term usage of antiglaucoma medicines.

\section{CONCLUSION}

The effects of antiglaucoma medications such as dry eye can influence compliance and success of treatment in glaucoma patients. From our study, we can conclude that patients on antiglaucoma therapy have a greater prevalence of ocular surface disease and dry eye. The causative factors were medications with preservatives and longer treatment duration.

\section{Acknowledgement: None}

Conflict of Interest: None

Source of Funding: None

Ethical Approval: Approved 


\section{REFERENCES}

1. Arici MK, Arici DS, Topalkara A, Güler C. Adverse effects of topical antiglaucoma drugs on the ocular surface. Clin Exp Ophthalmol. 2000;20(8):113-7.

2. Malvitte L, Montange T, Vejux A, Baudouin C, Bron AM, Creuzot-Garcher C, et al. Measurement of inflammatory cytokines by multicytokine assay in tears of patients with glaucoma topically treated with chronic drugs. $\mathrm{Br} \mathrm{J}$ Ophthalmol. 2007;91(1):29-32.

3. Baudouin C, Pisella PJ, Fillacier K, Goldschild M, Becquet F, De Saint Jean M, et al. Ocular surface inflammatory changes induced by topical antiglaucoma drugs: Human and animal studies. Ophthalmology. 1999;106(3):556-63.

4. Aritürk N, Öge I, Baris S, Erkan D, Süllü Y, Koc F. The effects of antiglaucomatous agents on conjunctiva used for various durations. In: International Ophthalmology. 20 (1). 1996. p. 57-62.

5. Foulks GN, Bron AJ. Meibomian gland dysfunction: A clinical scheme for description, diagnosis, classification, and grading. Ocul Surf. 2003;1(3):107-26.
6. Zhou S, Li Y, Lu ATH, Liu P, Tang M, Yiu $\mathrm{SC}$, et al. Reproducibility of tear meniscus measurement by Fourier-domain optical coherence tomography: A pilot study. Ophthalmic Surg Lasers Imaging. 2009;40(5):442-7.

7. Champeau EJ, Edelhauser HF. Effect of ophthalmic preservatives on the ocular surface: conjunctival and corneal uptake and distribution of benzalkonium chloride and chlorhexidine digluconate. In: Holly F, Lamberts D, Mac Keen D, editors. The preocular tear film in health, disease, and contact lens wear. Vol. 998. Lubbock, Texas: Dry Eye Institute Inc; pp. 292-302

8. De Saint Jean M, Debbasch C, Brignole F, Rat P, Warnet J-M, Baudouin C. Toxicity of preserved and unpreserved antiglaucoma topical drugs in an in vitro model of conjunctival cells. Curr Eye Res. 2000; 20(2):85-94.

How to cite this article: Ingle SY, Biswas S. Prevalence of ocular surface disorder and dry eye in patients with glaucoma using topical antiglaucoma medications. International Journal of Research and Review. 2021; 8(9): 912. DOI: https://doi.org/10.52403/ijrr.20210902 